\title{
The EQ-5D-5L in patients admitted to a hospital in Japan with recent spinal cord injury: a descriptive study
}

\author{
Ryuichiro Koga ${ }^{1} \cdot$ Takayuki Sasaki $^{1} \cdot$ Ryosuke Ideta $^{1} \cdot$ Kenji Iwahashi $^{1} \cdot$ Chinaru Tsugami $^{1} \cdot$ Akira Nishimura $^{1}$. \\ Tetsuo Hayashi ${ }^{1,2} \cdot$ Hiroaki Sakai $^{2} \cdot$ Takeshi Maeda $^{2}$
}

Received: 19 October 2018 / Revised: 24 May 2019 / Accepted: 24 May 2019 / Published online: 14 June 2019

(c) The Author(s), under exclusive licence to International Spinal Cord Society 2019

\begin{abstract}
Study design Descriptive study.

Objective To demonstrate Euro Quality of Life 5-dimensional 5-level (EQ-5D-5L) by severity level at the acute stage and discharge in patients with traumatic spinal cord injury (SCI).

Setting Spinal Injuries Center, Fukuoka, Japan.

Methods Patients with traumatic SCI who completed the EQ-5D-5L instrument at the acute stage and discharge were divided into four groups according to severity (severity group G1; C1-C4 ASIA Impairment Scale (AIS) A, B, and C, G2; C5-C8 AIS A, B, and C, G3; T1-S5 AIS A, B, and C, G4; all AIS D). All data were extracted from the Japan single-center study for spinal cord injury database (JSSCI-DB).

Results Data were collected from 139 individuals at the acute stage and 164 individuals at discharge. In the comparison of utility score by severity in acute stage and discharge, G3 was significantly higher in discharge ( 0.325 versus 0.580$)$. Utility scores by degree of severity were significantly higher in group G4 than those of G1, G2, and G3 at the acute stage. Utility scores at discharge were significantly higher in groups G3 and G4 than in G1, and there was a significant difference between groups G4 and G2.

Conclusions At each stage, the utility scores of group G4 were significantly higher than those of groups G1 and G2. Altogether, the utility scores for hospitalized patients with traumatic SCI that were indicated in this study will serve as basic data that can be used while performing spinal regeneration medical procedures in the future.
\end{abstract}

\section{Introduction}

The Euro Quality of Life 5-dimensional 5-level (EQ-5D-5L) is a comprehensive assessment instrument that was developed by the EuroQoL Group for the purpose of measuring health-related quality of life (HRQOL). It is widely employed in areas such as clinical research, research related to medical policy, and clinical trials [1-3]. One of its major characteristics is its ability to produce a utility score that can

Ryuichiro Koga

reha@sekisonh.johas.go.jp

1 Department of Rehabilitation Medicine, Japan Organization of Occupational Health and Safety, Spinal Injuries Center, Fukuoka, Japan

2 Department of Orthopedic Surgery, Japan Organization of Occupational Health and Safety, Spinal Injuries Center, Fukuoka, Japan be used to calculate quality-adjusted life years, and its standard values in the general population for a variety of illnesses are used throughout the world [4, 5]. Although there have been reports on the recovery of motor function and level of independence in activities of daily living (ADL) following rehabilitation interventions for patients with spinal cord injury (SCI), there have been no reports on health economics research in Japan [6, 7]. Thus, it was necessary to accumulate utility scores from an SCI population to serve as basic data. In addition, based on reports that the motor score of the key muscles, Walking Index for Spinal Cord Injury version II (WISCI II), and Spinal Cord Independence Measure version III (SCIM III) exhibit different improvement patterns depending on the severity level according to measures such as the level of the lesion and ASIA Impairment Scale (AIS), we hypothesized that utility scores would also differ depending on the timing and the level of severity [8].

The objective of this study was to demonstrate EQ-5D$5 \mathrm{~L}$ by severity level at the acute stage and discharge in 
patients with SCI who underwent inpatient rehabilitation at our hospital.

\section{Methods}

\section{Summary of the Japan single-center study for spinal cord injury database (JSSCI-DB)}

Our hospital is a specialized SCI center that provides early surgical treatment and integrated rehabilitation from the acute stage to the recovery stage in Japan. Therefore, this institution is able to compile baseline data that includes post-SCI neurological recovery and progress in patient ability to engage in the ADL. Since 2005, when the JSSCIDB was launched, a total of 1064 cases have been registered (as of October 2018). The JSSCI-DB contains outcome data of 134 items consisting of a neurological evaluation (e.g., AIS and neurological level of injury (NLI)), physical function evaluation (e.g., WISCI II and SCIM III), and an HRQOL evaluation (EQ-5D-5L). The EQ-5D-5L was added in February 2015.

\section{Summary of EQ-5D-5L}

The EQ-5D-5L essentially consists of two pages of the EQ-5D descriptive system and the EQ visual analog scale (EQ VAS) [9]. The EQ-5D-5L description system includes the following five dimensions: mobility, selfcare, usual activities, pain/discomfort, and anxiety/ depression. Furthermore, each dimension has the following five levels: no problems, slight problems, moderate problems, serious problems, and extreme problems. A unique health state is defined by combining one level from each of the five dimensions. A total of 3125 possible health states are defined in this way. Each state is referred to in terms of a 5-digit code. For example, state 11111 indicates no problems in any of the five dimensions, while state 12345 indicates no problems with mobility, slight problems with washing or dressing, moderate problems with doing usual activities, severe pain or discomfort, and extreme anxiety or depression. In addition, the EQ-5D-5L state can be converted into an utility score. The lowest utility score in the Japanese version of EQ-5D-5L is -0.025 [10]. The EQ VAS evaluates the respondent's self-rated health on a 20 -cm vertical, visual analog scale with endpoints labeled 'the best health you can imagine' and "the worst health you can imagine". This information can be used as a quantitative measure of health as judged by the individual respondents. The rights for the use of the EQ-5D-5L Japanese language version in this study were obtained by submitting the protocol for this study to the EuroQol Group's administrative office and subsequently receiving approval $\left({ }^{\circledR} 1990\right.$ EuroQol Group; EQ-5DTM is a trademark of the EuroQol Group).

\section{Participants}

The participants were patients with traumatic SCI registered in the JSSCI-DB and who underwent inpatient rehabilitation at the Spinal Cord Injuries Center in Fukuoka Prefecture, Japan, between February 2015 and January 2018. We extracted data related to the participants' age, sex, length of stay (LoS), and AIS, NLI, and EQ-5D-5L at the acute stage ( 2 weeks post injury) and at discharge from the JSSCI-DB. Those with an AIS score of E (normal), those from whom consent to participate in this study was not obtained, those who revoked said consent, and those who had diseases that could affect HRQOL (e.g., severe heart disease, uncontrolled diabetes, cancer, psychiatric disorders, and cognitive disorders) were excluded. In this study "acute stage" was defined as the time point 2 weeks after injury (time of initial EQ-5D-5L survey) and "discharge" was defined as the time point at which inpatient rehabilitation was completed. The participants were divided into four groups by the level of severity using the NLI, which is based on the ISNCSCI, and the AIS (severity group G1; C1-C4 AIS A, B, and C, severity group G2; C5-C8 AIS A, $\mathrm{B}$, and $\mathrm{C}$, severity group G3; T1-S5 AIS A, B, and C, severity group G4; All AIS D) [11].

\section{Survey method}

The survey method utilized with the EQ-5D-5L is, as a general rule, a self-administered questionnaire format that is completed by each person in his or her own hospital room in order to prevent any outside information influence. In cases in which upper extremity paralysis made it difficult for a participant to complete the form on his or her own, a QOL survey team member asked the questions and indicated the answers on the form for the participant.

\section{Statistical and analytical methods}

For the health profile we assessed the problem ratios in each variable category at the acute stage and at discharge. Furthermore, we compared the utility scores at each level of severity at the acute stage and at the discharge using the Mann-Whitney $U$ test. We also analyzed the utility scores by the level of severity for each time point using the Kruskal-Wallis test. Analysis was performed using MATLAB (Math Works, Natick, MA, USA). The standard of statistical significance was set at $5 \%$. If not mentioned otherwise, results including numbers in figures and tables are presented as median and interquartile range (IQR) in brackets. 
Table 1 Characteristics of the participants

\begin{tabular}{lcc}
\hline & $\begin{array}{l}\text { Acute stage } \\
n=139\end{array}$ & $\begin{array}{l}\text { Discharge } \\
n=164\end{array}$ \\
\hline $\begin{array}{l}\text { Age (years) } \\
\text { Median (IQR) }\end{array}$ & $66(58-74)$ & $63(55-73)$ \\
Gender & & \\
$\quad$ Male, $n(\%)$ & $111(79.9)$ & $31(18.9)$ \\
Female, $n(\%)$ & $28(20.1)$ & \\
LoS (days) & & $241(126-351)$ \\
Median (IQR) & & $39(23.8)$ \\
NLI and AIS & $58(41.8)$ & $21(12.8)$ \\
G1, $n(\%)$ & $23(16.5)$ & $22(13.4)$ \\
G2, $n(\%)$ & $12(8.6)$ & $82(50.0)$ \\
G3, $n(\%)$ & $46(33.1)$ & $50(23-78)$ \\
G4, $n(\%)$ & & $60(30-80)$ \\
EQ VAS & $50(20-52)$ & $70(66-80)$ \\
G1, median (IQR) & $30(10-55)$ & $70(50-80)$ \\
G2, median (IQR) & $28(24-50)$ & $63(50-80)$ \\
G3, median (IQR) & & \\
G4, median (IQR) & & \\
\hline
\end{tabular}

G1, C1-4 AIS A, B, and C; G2, C5-8 AIS A, B, and C; G3, T1-S5 AIS A, B, and C; G4, All AIS D

$I Q R$ interquartile range, $L o S$ length of stay, $N L I$ neurological level of injury, AIS ASIA (American Spinal Injury Association) Impairment Scale, EQ VAS the EQ Visual Analog Scale

\section{Results}

Final valid data were collected from 139 individuals at the acute stage and from 164 individuals at discharge. The median age at the acute stage and at discharge was 66 years and 63 years, respectively, and $80 \%$ of the participants were males. The median LoS at discharge was 241 days (IQR 126-351 days). Table 1 shows the basic characteristics and EQ VAS for all participants at both time points.

\section{EQ-5D-5L health profiles}

The percentage of participants who responded "no problems" for all categories was $2.9 \%$ at the acute stage and $3.6 \%$ at discharge, the majority of which were in group G4. The percentage of those with the lowest possible utility score of -0.025 was $0.7 \%$ at the acute stage and $0.6 \%$ at discharge, the majority of which were in group G1. Investigation of mobility, self-care, and usual activities at the acute stage pointed out that there was a high percentage of participants in groups G1, G2, and G3 with problems, and that many participants in group G3 reported anxiety/ depression. At discharge, participants in group G1 were more likely to have problems in all dimensions than those in the other groups. Among participants in group G1, those at discharge had more problems in all dimensions than those in the acute stage (Fig. 1).

\section{EQ-5D-5L utility score}

The utility scores at each level of severity, at the acute stage, and at discharge were compared and found to be significantly higher at discharge in group G3 $(p=0.021)$. There were no significant differences in the other groups. In group G1, the utility score decreased from 0.317 at the acute stage to 0.290 at discharge (Table 2). Investigation of utility scores by the level of severity at each time point indicated that the utility scores at the acute stage were significantly higher in group G4 than in groups G1, G2, and G3 $(p<0.05)$. Investigation of utility scores at discharge revealed that group G3 had a significantly higher score than group G1 $(p<0.05)$, and that the score for group G4 was significantly different compared with groups G1 and G2 $(p<0.001)$ (Table 3).

\section{Discussion}

This study elucidated details on the changes in the subjectively assessed health status of patients in the initial treatment stage following traumatic SCI by the level of severity according to international assessment classifications. This is the first report in Japan using the EQ-5D-5L in patients with traumatic SCI who underwent consistent rehabilitation therapy from the acute stage to discharge.

Using the EQ-5D-5L health profile, we identified the percentages of participants who reported problems in each variable category. A higher percentage of problems in groups G1 and G2 in both periods were reported with respect to mobility, self-care, and usual activities, rather than pain/discomfort and anxiety/depression. This suggests that the influence of tetraplegia may lead to limited mobility and problems in usual activities. Furthermore, comparison of the two time points indicated that there were significant differences in the utility scores by the level of severity only in group G3 (close to paraplegia). It is possible that this result was due to the fact that paralysis had little effect in group G3 on the upper extremities, and as a result, the percentage of participants who felt they had problems with the ADL decreased at discharge. This can be confirmed using the health profiles, and we found that the percentage of problems with items related to self-care was lower in group G3 than in groups G1 and G2. In contrast, only the utility score in group G1, which is the classification for patients with high cervical spinal cord injuries, decreased. Investigation of health profiles suggested that for all variable categories, group G1 reported higher percentages of problems compared with the other groups. In this study, the utility scores at both 


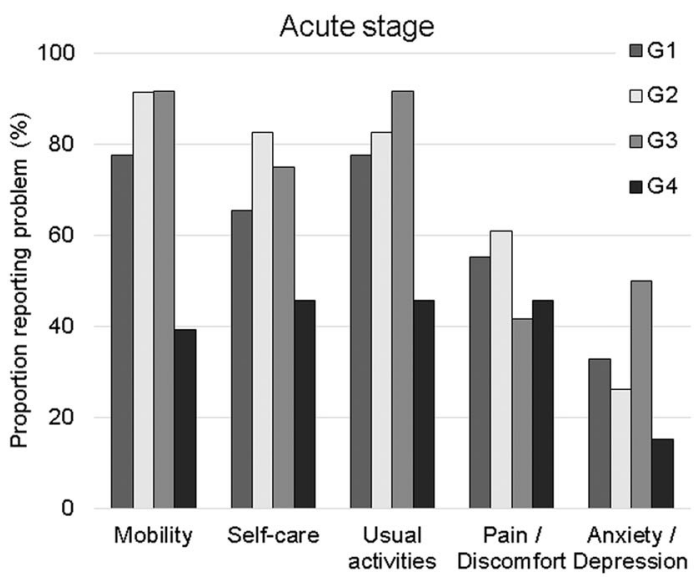

Fig. 1 Profile with problems with each variable category of EQ-5D$5 \mathrm{~L}$. The figure shows the percentages of problems rated from "moderate problems" to "extreme problems" (i.e., Levels 3-5) as reported

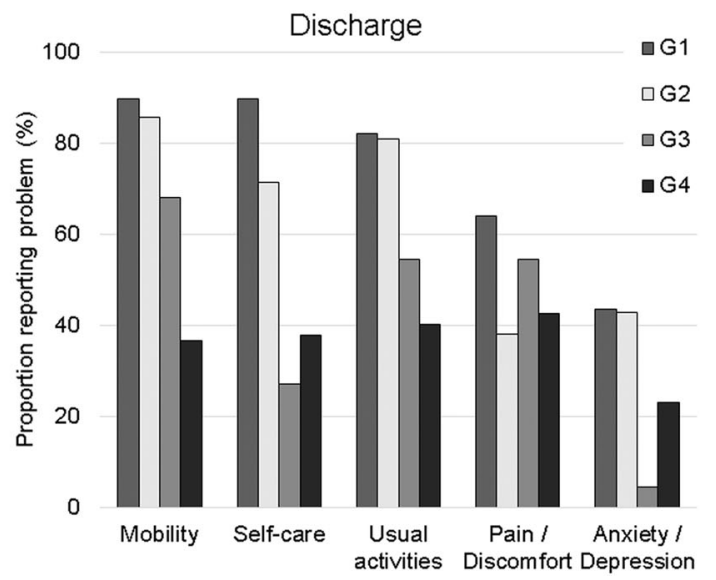

by the participants for each item category of the EQ-5D-5L by the level of severity at the acute stage and at discharge
Table 2 Comparative analysis of utility score in acute stage and discharge in each group

Table 3 Analysis of utility score for each group during acute stage and discharge

\begin{tabular}{llll}
\hline & Utility score & & $P$-value \\
\cline { 2 - 3 } & Acute stage & Discharge & \\
\hline G1, median (IQR) & $0.317(0.222-0.532)$ & $0.290(0.171-0.364)$ & 0.150 \\
G2, median (IQR) & $0.317(0.191-0.413)$ & $0.369(0.229-0.489)$ & 0.565 \\
G3, median (IQR) & $0.325(0.265-0.364)$ & $0.580(0.414-0.661)$ & 0.021 \\
G4, median (IQR) & $0.661(0.358-0.797)$ & $0.665(0.419-0.806)$ & 0.673 \\
\hline
\end{tabular}

G1, C1-4 AIS A, B, and C; G2, C5-8 AIS A, B, and C; G3, T1-S5 AIS A, B, and C; G4, All AIS D $I Q R$ interquartile range

$* P<0.05$ versus discharge

\begin{tabular}{lllll}
\hline & Utility score & & \\
\cline { 2 - 5 } & G1 & G2 & G3 & G4 \\
\hline Acute stage & $0.317(0.222-0.532)^{*}$ & $0.317(0.191-0.413)^{*}$ & $0.325(0.265-0.364)^{*}$ & $0.661(0.358-0.797)$ \\
Discharge & $0.290(0.171-0.364)^{\dagger},+$ & $0.369(0.229-0.489)^{\ddagger}$ & $0.580(0.414-0.661)$ & $0.665(0.419-0.806)$ \\
\hline
\end{tabular}

G1, C1-4 AIS A, B, and C; G2, C5-8 AIS A, B, and C; G3, T1-S5 AIS A, B, and C; G4, All AIS D

Data are presented as group median (interquartile range)

$* P<0.05$ versus $\mathrm{G} 4$

${ }^{\dagger} P<0.05$ versus $\mathrm{G} 3$

${ }^{\ddagger} P<0.001$ versus G4

time points were highest in group G4. Those with an AIS score of D in group $\mathrm{G} 4$ have a high probability of regaining the ability to walk. It is likely that this is the reason that they reported fewer problems with mobility, self-care, and usual activities compared with the other groups [12-15].

These results support the findings by Rivers et al. that severe injury and health conditions are factors affecting HRQOL, and those of a similar study by Richard-Denis et al. on patients with subacute cervical traumatic SCI $[16,17]$. Furthermore, Whitehurst et al. [18] reported that the median utility scores for patients with chronic SCI registered to the Canada-wide SCI research project-the
SCI Community Survey-were 0.465 for those who were unable to walk and 0.579 for those who were able to walk. This result suggests that the walking ability is important with regards to the utility score.

To summarize the above, in the acute stage utility scores were higher in group $\mathrm{G} 4$, which had good voluntary muscle strength, and at discharge, they were higher in groups G3 and G4, which were more likely to acquire ADL, than in groups G1 and G2. Going forward, clinical approaches that use assistive devices (such as orthoses and robots) to increase the amount G1 and G2 patients are capable of doing on their own could help to increase utility scores. 
This study had several limitations. First, there were differences in the timing of discharge and LoS among the participants. In order to solve this problem, we should have set additional conditions such as post-injury follow-up period and post-discharge-to-home. In addition, although we utilized the EQ-5D-5L, which is a comprehensive scale, it is difficult to ascertain detailed changes in patient's condition when using this scale, as it contains a small number of items and is a simplified scale. Thus, it remains necessary to conduct a further study on this issue using a combination of disease-specific scales. Such a method is also recommended by cost-effectiveness studies [19]. In the field of SCI, it is important to conduct cost-effectiveness studies in order to determine the effectiveness of medical interventions. In order to do this, the most urgent problem is the collection of utility score data for a variety of patient statuses. This, we believe, is the first step in a cost-effectiveness study. In the present study, we conducted a very simple investigation in which we analyzed patients with traumatic SCI by the level of severity using the EQ-5D-5L. In the future, we would like to analyze the treatment effect through intervention studies such as randomized controlled trials.

We believe the utility scores for the hospitalized patients with traumatic SCI that were indicated in this study will serve to provide basic data that we can draw upon for performing spinal regeneration medical techniques in the future.

\section{Data archiving}

All relevant data are within this manuscript and raw data are archived by the authors. The datasets generated during and/ or analyzed during the current study are not publicly available due to including private information of patients but are available from the corresponding author on reasonable request.

Acknowledgements We would like to express their deep appreciation to Fumio Tsutsumi (formerly of the Department of Rehabilitation Medicine, Kyushu Nutrition Welfare University), who provided valuable advice regarding the statistical analysis conducted in this study. The authors would also like to express their appreciation to Prof. Shinichi Noto (Department of Medical Technology, Niigata University of Health and Welfare) for his kind cooperation in this study.

Author contributions RK was the principal author of this paper and wrote the summary of the study, collected the data, and wrote the manuscript. TS extracted the data, conducted the statistical analysis, and created Fig. 1 and Tables $1-3$. RI provided the registry data. KI and CT collected the data used in this study and screened the participants. RI, TS, KI, and CT screened the protocol, analyzed the results, critiqued the manuscript, made revisions, and provided the author with support when writing the final draft of this paper. AN, TH, HS, and TM provided feedback on the paper. All authors approved the final draft.

\section{Compliance with ethical standards}

Conflict of interest The authors declare that they have no conflict of interest.

Ethics This study was approved by the Institutional Review Board of our hospital. Both written and oral explanations of the contents of this study were provided to the participants and their consent was obtained.

Publisher's note: Springer Nature remains neutral with regard to jurisdictional claims in published maps and institutional affiliations.

\section{References}

1. Azuma MK, Ikeda S. Investigation of evidence sources for healthrelated quality of life in cost-utility analysis of pharmaceuticals in Japan. Value Health Reg Issues. 2014;3:190-6.

2. Brauer CA, Rosen AB, Greenberg D, Neumann PJ. Trends in the measurement of health utilities in published cost-utility analyses. Value Health. 2006;9:213-8.

3. Polinder S, van Beeck EF, Essink-Bot ML, Toet H, Looman CW, Mulder S, et al. Functional outcome at 2.5, 5, 9, and 24 months after injury in The Netherlands. J Trauma. 2007;62:133-41.

4. Arora M, Harvey LA, Glinsky JV, Chhabra HS, Hossain MS, Arumugam N, et al. Cost-effectiveness analysis of telephonebased support for the management of pressure ulcers in people with spinal cord injury in India and Bangladesh. Spinal Cord. 2017;55:1071-8.

5. Nagayama H, Tomori K, Ohno K, Takahashi K, Nagatani R, Izumi $\mathrm{R}$, et al. Cost effectiveness of the occupation-based approach for subacute stroke patients: result of a randomized controlled trial. Top Stroke Rehabil. 2017;24:337-44.

6. Fawcett JW, Curt A, Steeves JD, Coleman WP, Tuszynski MH, Lammertse D, et al. Guidelines for the conduct of clinical trials for spinal cord injury as developed by the ICCP panel: spontaneous recovery after spinal cord injury and statistical power needed for therapeutic clinical trials. Spinal Cord. 2007;45: 190-205.

7. Whitehurst DG, Mittmann N. The value of health economics research in spinal cord injury. Spinal Cord. 2013;51:586-7.

8. van Hedel HJ. Improvement in function after spinal cord injury: the black-box entitled rehabilitation. Swiss Med Wkly. 2012;142: w13673.

9. Reenen MV, Janssen B. EQ-5D-5L user guide: basic information on how to use the EQ-5D-5L instrument. 2015. https://euroqol. org/wp-content/uploads/2016/09/EQ-5D-5L_UserGuide_2015.pdf.

10. Ikeda S, Shiroiwa T, Igarashi A, Noto S, Fukuda T, Saito S, et al. Developing a Japanese version of the EQ-5D-5L value set. J Natl Inst Public Health. 2015;64:47-55.

11. Kirshblum SC, Burns SP, Biering-Sorensen F, Donovan W, Graves DE, Jha A, et al. International standards for neurological classification of spinal cord injury (revised 2011). J Spinal Cord Med. 2011;34:535-46.

12. New PW. Functional outcomes and disability after nontraumatic spinal cord injury rehabilitation: results from a retrospective study. Arch Phys Med Rehabil. 2005;86:250-61.

13. Scivoletto G, Morganti B, Ditunno P, Ditunno JF, Molinari M. Effects on age on spinal cord lesion patients' rehabilitation. Spinal Cord. 2003;41:457-64.

14. Burns SP, Golding DG, Rolle WA Jr, Graziani V, Ditunno JF Jr. Recovery of ambulation in motor-incomplete tetraplegia. Arch Phys Med Rehabil. 1997;78:1169-72. 
15. Kay ED, Deutsch A, Wuermser LA. Predicting walking at discharge from inpatient rehabilitation after a traumatic spinal cord injury. Arch Phys Med Rehabil. 2007;88:745-50.

16. Rivers CS, Fallah N, Noonan VK, Whitehurst DG, Schwartz CE, Finkelstein JA, et al. Health conditions: effect on function, healthrelated quality of life, and life satisfaction after traumatic spinal cord injury. A prospective observational registry cohort study. Arch Phys Med Rehabil. 2018;99:443-51.

17. Richard-Denis A, Thompson C, Mac-Thiong JM. Quality of life in the subacute period following a cervical traumatic spinal cord injury based on the initial severity of the injury: a prospective cohort study. Spinal Cord. 2018;56:1042-50.

18. Whitehurst DGT, Mittmann N, Noonan VK, Dvorak MF, Bryan S. Health state descriptions, valuations and individuals' capacity to walk: a comparative evaluation of preference-based instruments in the context of spinal cord injury. Qual Life Res. 2016;25:2481-96.

19. Haywood KL, Wilson R, Staniszewska S, Salek S. Using PROMs in healthcare: who should be in the driving seat-policy makers, health professionals, methodologists or patients? Patient. 2016;9: 495-8. 